Pacific Journal of Mathematic 


\section{RECONSTRUCTING INFINITE GRAPHS}

\section{J. A. Bondy AND R. L. Hemminger}

It is a well-known conjecture of $S$. M. Ulam that any finite graph of order at least three can be reconstructed from its maximal vertex-deleted subgraphs. Formally (writing $G_{v}$ for $G-v$ ) Ulam's Conjecture states: if $G$ and $H$ are finite graphs of order at least three such that there is a bijection $\sigma: V(G) \rightarrow V(H)$ with the property

$$
G_{v} \cong H_{o(v)} \text { for all } v \in V(G),
$$

then $G \cong H$. This conjecture has not been proved in general, although it was shown by $P$. J. Kelly to be true for disconnected graphs and trees and has also been verified for several other classes of graphs. The purpose of this paper is to examine Ulam's Conjecture for infinite graphs. (It is trivial to determine, from any $G_{v}$, whether or not a graph $G$ is infinite.) Results are obtained which can loosely be viewed as extensions of Kelly's work on disconnected graphs and trees.

In $\S 2$ it is shown that infinite graphs $G$ and $H$ satisfying (1) must have the same finite components, occurring with the same multiplicity. Corollaries of this are that if $G$ either has only finite components, or has some finitely occurring finite component, then $G \cong H$. In $\S 3$ the conjecture is proved for $m$-coherent locally finite trees, where $m$ is finite and greater than one. This furnishes a partial solution to the reconstruction problem for infinite trees, raised by C. St. J. A. Nash-Williams.

We have used the language of reconstruction in our proofs. However, it should be noted that the results are existential in nature and not algorithmic. Throughout the paper $G$ and $H$ will denote infinite graphs satisfying condition (1) of Ulam's Conjecture. Any notation and terminology not defined can be found in Harary [3].

2. Disconnected graphs. We denote by $c(G)$ the number of components of $G$, and by $c(G ; K)$ the number of components of $G$ that are isomorphic to $K$. A finite connected graph $J$ is called a $K$-producer if $c\left(J_{v} ; K\right)>0$ for all $v \in V(J)$. (Since $J$ has a non-cutvertex, $J$ must be regular and of order one more than the order of $K$; hence $K$ determines $J$ up to isomorphism.) An endvertex of $G$ is a vertex of degree one.

LEMMA 2.1. If $L$ is infinite and connected and $K$ is finite, then there is an infinite set $S \subseteq V(L)$ such that $c\left(L_{v} ; K\right)=0$ for all $v \in S$. 
Proof. Let $T$ be a spanning tree of $L$. If $T$ has an infinite number of endvertices, let $S$ be the set of endvertices of $T$. If $T$ has no endvertices, any infinite subset $S$ of $L$ has the required property. If $T$ has a finite positive number of endvertices let $w_{1}$ be one of these and let $\left(w_{1}, w_{2}, w_{3}, \cdots\right)$ be a one-way infinite path in $T$ from $w_{1}$. Since $T$ has a finite number of endvertices there is an $N$ such that, for $n>N$, the components of $T-w_{n}$ all have order greater than the order of $K$. The set $S=\left\{w_{i}: i>N\right\}$ has the required property.

Lemma 2.2. G has a component of cardinality at least $\boldsymbol{\aleph}_{2}$ if and only if some $G_{v}$ has a component of cardinality at least $\boldsymbol{\aleph}$. .

Proof. If $G$ has a component of cardinality at least $\boldsymbol{\aleph}_{*}$, then either there is a vertex $v$ whose degree has cardinality less than $\aleph_{*}$, in which case some component of $G_{v}$ has cardinality at least $\boldsymbol{\aleph}_{\nu}$, or else the degree of every vertex of $G$ is of cardinality at least $\aleph$. and then every component of every $G_{v}$ has cardinality at least $\boldsymbol{\aleph}_{\nu}$. The converse is obvious.

LEMma 2.3. The component orders of $G$ have a finite upper bound if and only if the component orders of every $G_{v}$ have a finite upper bound.

Proof. By Lemma 2.2 we can assume that $G$ has only components of finite order. The result is then clear.

Lemma 2.4. For $K$ finite, $c(G ; K)=\infty$ if and only if $c\left(G_{v} ; K\right)=$ $\infty$ for all $v \in V(G)$.

Proof. The condition is obviously necessary. Conversely, suppose $c(G ; K)<\infty$. For any finite component $C$ of $G$, clearly $c\left(C_{v} ; K\right)<\infty$. Therefore, if $G$ has only finite components, $c\left(G_{v} ; K\right)<\infty$. If $G$ has an infinite component $L$ then, by Lemma $2.1, c\left(G_{v} ; K\right)=c(G ; K)<\infty$ for some $v \in V(G)$.

The next lemma shows that we can distinguish whether or not $c(G ; K)$ is positive when $K$ is finite.

Lemma 2.5. Let $K$ be a finite connected graph. Then

(a) if the component orders of $G$ have no finite upper bound, $c(G ; K)>0$ if and only if $c\left(G_{v} ; K\right)>0$ for all but finitely many $v$;

(b) if the component orders of $G$ have a finite upper bound and every infinitely occurring component is a $K$-producer, $c(G ; K)>0$ if 
and only if $c\left(G_{v} ; K\right)>1$ for infinitely many $v$;

(c) if the component orders of $G$ have a finite upper bound and not every infinitely occurring component is a K-producer, $c(G ; K)>$ 0 if and only if $c\left(G_{v} ; K\right)=0$ for at most $|V(K)|$ vertices $v$.

Proof. By Lemmas 2.3 and 2.4 we can distinguish between cases (a), (b), and (c). (By Lemma 2.4 we can determine, from the $G_{v}$, the infinitely occurring finite components-one examines these for $K$-producers.)

(a) If $c(G ; K)>0$ then $c\left(G_{v} ; K\right)>0$ for all $v \notin V(K)$. Conversely suppose that $c(G ; K)=0$. By Lemma 2.2 we can distinguish between

(i) $G$ has a component $L$ of infinite order; or

(ii) every component of $G$ has finite order.

(i) By Lemma 2.1, $c\left(L_{v} ; K\right)=0$ for infinitely many $v \in V(L)$, and hence $c\left(G_{v} ; K\right)=0$ for infinitely many $v \in V(G)$.

(ii) For each non-cut-vertex $v$ of a component of $G$ of order greater than $|V(K)|+1, c\left(G_{v} ; K\right)=0$. There are infinitely many such components and each of them contains at least two non-cutvertices.

(b) Since, for each $K$-producer $J$ and each $v \in V(J), c\left(J_{v} ; K\right)=1$, $c(G ; K)>0$ implies $c\left(G_{v} ; K\right)>1$ for infinitely many $v$. Conversely suppose that $c(G ; K)=0$. Since the component orders of $G$ have a finite upper bound, there are finitely many isomorphism types of components of $G$. It follows that there are finitely many components which are not infinitely occurring. Since $c\left(G_{v} ; K\right)=1$ when $v$ is in a $K$-producer, $c\left(G_{v} ; K\right)$ can be greater than one only for finitely many $v$.

(c) If $c(G ; K)>0$ and if $C$ is a component of $G$ with $C \cong K$, then $c\left(G_{v} ; K\right)>0$ for all $v \notin V(C)$, and so $c\left(G_{v} ; K\right)=0$ for at most $|V(K)|$ vertices $v$. Conversely suppose that $c(G ; K)=0$ and let $C_{1}$, $C_{2}, \cdots$ be components of $G$ with $C_{i} \cong J$ for all $i$, where $J$ is not a $K$-producer. Then there exist vertices $v_{1} \in V\left(C_{1}\right), v_{2} \in V\left(C_{2}\right), \cdots$ such that $c\left(G_{v_{i}} ; K\right)=0$ for all $i$. That is, $c\left(G_{v} ; K\right)=0$ for infinitely many vertices $v$.

Lemma 2.6. Let $K$ be a finite connected graph such that $c(G ; K)>$ 0. Then

$$
c(G ; K)=1+\min _{v}\left\{c\left(G_{v} ; K\right)\right\}
$$

Proof. If $v \in V\left(K^{\prime}\right)$ where $K^{\prime}$ is a component of $G$ with $K^{\prime} \cong K$, then $c\left(G_{v} ; K\right)=c(G, K)-1$. Otherwise $c\left(G_{v} ; K\right) \geqq c(G ; K)$.

THEOREM 1. Let $G$ and $H$ be infinite graphs satisfying (1). If $G$ is disconnected and either 
(a) every component of $G$ has finite order, or

(b) $G$ has a finite component $K$ which is isomorphic to only finitely many components of $G$, then $G \cong H$.

Proof. By Lemmas 2.5 and 2.6, $G$ and $H$ have the same finite components for any disconnected graph $G$. Note that Lemma 2.6 holds even when $c(G ; K)=\infty$.

To show that $G$ and $H$ have the same infinite components in case (b) we note that if $c\left(G_{v} ; K\right)=c(G ; K)-1$, then $v \in V\left(K^{\prime}\right)$ for some component $K^{\prime}$ of $G$, with $K^{\prime} \cong K$. For such a $v$, the infinite components of $G$ are the same as the infinite components of $G_{v}$.

The finiteness conditions in the theorem suggest that one might hope to prove $G \cong H$ if $1<c(G)<\infty$. However, a counterexample due to J. Fisher, R. L. Graham, F. Harary, and J. A. B. Zonker [2] shows that this is not possible. Let $T$ be a tree in which each vertex has degree $\boldsymbol{\aleph}_{0}$, and let $G$ and $H$ be forests in which each component is isomorphic to $T$. If $G$ has $k$ such components and $H$ has $l$ such components, where $1 \leqq k<l \leqq \boldsymbol{\aleph}_{0}$, then $G$ and $H$ satisfy the hypotheses of Ulam's Conjecture, but clearly $G \not H$.

3. Locally finite trees. Since $G$ is not a cycle (it is infinite) $G$ is a forest if and only if $G_{v}$ is a forest for all $v \in V(G)$. Theorem 1 shows that we can reconstruct many types of forest. However, since $G$ and $H$ are forests in the counterexample mentioned at the end of $\S 2$, we can not always reconstruct a forest; moreover, the counterexample shows that we can not even determine the number of components of $G$. Thus some restrictions are needed if we are to make further progress. In light of the counterexample, and since $G$ is locally finite if and only if $G_{v}$ is locally finite for all $v \in V(G)$, the property of being locally finite is a natural restriction to impose. In the remainder of this section $G$ will always denote a locally finite graph. Consequently we have

Lemma 3.1. $c(G)=\boldsymbol{\aleph}_{\nu}$ if and only if $c\left(G_{v}\right)=\boldsymbol{\aleph}_{\nu}$ for all $v \in V(G)$.

Denote by $d(v)$ the degree of vertex $v$ in $G$ and by $\delta(G)$ the minimum vertex degree in $G$. By Lemma 2.5 we can distinguish whether or not $\delta(G)=0$, and by Lemma 3.1 whether or not $c(G)$ is finite.

Lemma 3.2. Let $G$ be a forest with $c(G)$ finite. Then

(a) if $\delta(G)=0$, then $c(G)=1+\min _{v}\left\{c\left(G_{v}\right)\right\}$;

(b) if $\delta(G)>0$, then $c(G)=\min _{v}\left\{c\left(G_{v}\right)\right\}-\min _{w}\left\{\delta\left(G_{w}\right)\right\}$, and in either case $d(v)=c\left(G_{v}\right)-c(G)+1$. 
Proof. Since $G$ is a locally finite forest

$$
c\left(G_{v}\right)=c(G)+d(v)-1<\infty .
$$

This proves the final assertion. It also follows that

$$
\min _{v}\left\{c\left(G_{v}\right)\right\}=c(G)+\delta(G)-1 \text {. }
$$

Putting $\delta(G)=0$ gives (a). To see (b) note that when $\delta(G)>0$, $\delta(G)=1+\min _{w}\left\{\delta\left(G_{w}\right)\right\}$.

Note. It is not possible in general to determine $d(v)$ for a particular $v$; for example, let $G=\infty K_{1} \cup \infty K_{2}$.

By Lemma 3.2, we know $c(G)$ and hence whether or not $G$ is a tree. Hereafter, we further restrict $G$ to be a locally finite tree. In this connection, we note that Harary, Schwenk, and Scott [4] have given counterexamples in which $G$ and $H$ are locally finite forests.

A tree is $m$-coherent if there are precisely $m$ distinct one-way infinite paths emanating from each $v \in V(G)$. A vertex $v$ is a base vertex of $G$ if $G_{v}$ has at least three components of infinite order, at least one of which is 1-coherent. $B$ is a branch of $G$ if $B$ is a 1coherent component of $G_{v}$ for some base vertex $v$; $v$ is a branch vertex if $v \in V(B)$ for some branch $B$.

LEMMA 3.3. An m-coherent tree, $m$ finite, has at most $m$ base vertices.

Proof. Let $B$ be a branch of $G$ associated with the base vertex $v$ (that is, $B$ is a 1-coherent component of $G_{v}$ ). Thus if $u \in V(B), w \in V(G)-V(B)$, and $(u, w) \in E(G)$ then $w=v$. It follows that a branch is associated with a unique base vertex. Moreover, for $u \in V(B), G_{u}$ has at most two components of infinite order. Thus branch vertices are not base vertices and it follows that branches of $G$ are vertex disjoint, since otherwise some vertex on two branches would be both a base vertex and a branch vertex. Thus an $m$-coherent tree, $m$ finite, has $m$ branches and therefore at most $m$ base vertices.

It can, in fact, be shown that, for $m>1$, an $m$-coherent tree has at most $m-2$ base vertices, but this stronger result is not needed here.

The cores of the proofs of Theorems 2 and 3 (to follow) rely on ones ability to identify (from the $G_{v}$ 's) a specific subgraph of the tree $G$. We shall in each case refer to this subgraph as the centre of $G$ even though the definition of the centre will vary, depending on the case being considered. 
Let $G$ be an $m$-coherent tree, $2<m<\infty$. Then, for use in Lemma 3.4 and Theorem 2, we define the centre of $G$ to be the subgraph of $G$ induced by the set of all nonbranch vertices of $G$; consequently the centre of $G$ is the subgraph of $G$ that is the union of all paths between base vertices of $G$ and all maximal trees rooted at vertices of these paths that contain no other vertices of these paths and no branch vertices. One easily sees that these maximal trees are finite.

Lemma 3.4. If $G$ is an m-coherent tree, $2<m<\infty$, then the centre of $G$ is finite and nonempty.

Proof. By Lemma 3.3, $G$ has at most $m$ base vertices. Thus, there are a finite number of paths between base vertices and each path is of finite length. Therefore, by the above observation, the centre of $G$ is finite.

Lemma 3.5. Let $G$ be a tree and let the components of some $G_{v}$ be $\left\{G_{i}\right\}_{\imath \in I}$ where $G_{i}$ is $m_{i}$-coherent. Let $m=\sum_{\imath \in I} m_{\imath}$. Then $G$ is $m$ coherent.

THEOREM 2. Let $G$ and $H$ be infinite graphs satisfying (1). If $G$ is an $m$-coherent locally finite tree, $2<m<\infty$, then $G \cong H$.

Proof. Lemma 3.5 ensures that we can determine the value of $m$ for which $G$ is $m$-coherent and hence whether or not $G$ is $m$-coherent for some finite $m>2$.

By Lemma 3.2 we can distinguish between three cases:

(a) $G$ has no endvertices;

(b) $G$ has a positive finite number of endvertices; or

(c) $G$ has infinitely many endvertices.

(a) In this case the branches of $G$ are paths and since, by Lemma 3.4, the centre of $G$ is finite there is a $v \in V(G)$ such that $G_{v}$ has two components each with exactly one endvertex. $G$ is obtained from $G_{v}$ by adding a vertex and joining it to these two endvertices.

(b) There is a $u \in V(G)$ such that $G_{u}$ has exactly two components, one of which is a one-way infinite path (implying that $u$ is a branch vertex). Let $d$ be the maximum distance of an endvertex in $G_{u}$ to a vertex of degree three or more in $G_{u}$.

Then there is a $v \in V(G)$ such that $G_{v}$ has exactly two components, one of which is a one-way infinite path and the other containing an endvertex at a distance greater than $d$ from any vertex of degree three or more. $G$ is obtained from $G_{v}$ by adding a vertex and joining it to the two endvertices. 
(c) If $L$ is an $m$-coherent locally finite tree, $2<m<\infty$, let $L^{k}$ denote the subgraph of $L$ that is the union of the centre of $L$ and all paths of length at most $k$ emanating from base vertices of $L$. Clearly, for each endvertex $v$ of $G,\left(G_{v}\right)^{k} \subseteq G^{k}$, and since $G$ has infinitely many endvertices, for each $k \geqq 1$, there is a $v_{k} \in V(G)$ such that $\left(G_{v_{k}}\right)^{k}$ has the same order as $G^{k}$. Therefore $G^{k}=\left(G_{v_{k}}\right)^{k}$, and similarly $H^{k}=\left(H_{\sigma\left(v_{k}\right)}\right)^{k}$. Also, since $G_{v_{k}} \cong H_{o\left(v_{k}\right)}$, there is an isomorphism of $\left(G_{v_{k}}\right)^{k}$ onto $\left(H_{\sigma\left(v_{k}\right)}\right)^{k}$ which maps the centre of $G_{v_{k}}$ onto the centre of $H_{\sigma\left(v_{k}\right)}$. It follows that, for $k=1,2,3, \cdots$, there exists an isomorphism of $G^{k}$ onto $H^{k}$ which maps the centre of $G$ onto the centre of $H$. Let $S_{k}$ denote the set of all such isomorphisms. If $y \in S_{k}$ and $x \in S_{k+1}$, and if $x$ restricted to $G^{k}$ is $y$, we write $y<x$. Since $S_{1}, S_{2}, S_{3}, \cdots$ is an infinite sequence of disjoint nonempty finite sets, it follows from König's Lemma (see, for example, [6], p. 288) that there exists an infinite sequence $x_{1}, x_{2}, x_{3}, \cdots$ such that $x_{k} \in S_{k}(k=$ $1,2,3, \cdots)$ and $x_{1}<x_{2}<x_{3}<\cdots$. This sequence defines an isomorphism of $G$ onto $H$.

THEOREM 3. Let $G$ and $H$ be infinite graphs satisfying (1). If $G$ is a 2-coherent locally finite tree, then $G \cong H$.

Proof. Lemmas 3.2 and 3.5 ensure that we can determine whether or not $G$ is a 2-coherent locally finite tree. By Lemma 3.2, $v$ is an endvertex in $G$ if and only if $G_{v}$ is connected.

(a) $G$ has finitely many endvertices.

If $G$ has no endvertex, $G$ is a two-way infinite path and so $G \cong$ $H$. Otherwise, we now define the centre of $G$ to be the subgraph of $G$ that is the union of all paths between endvertices of $G$. Since $G$ has only finitely many endvertices this centre is finite. The proof in this case is analogous to that used for case (b) of Theorem 2.

(b) $G$ has infinitely many endvertices.

Let $K$ be a 2-coherent graph with two-way infinite path $(\cdots$, $-2,-1,0,1,2, \cdots)$. The maximal connected subgraph of $K$ rooted at $m$ that does not contain $m-1$ or $m+1$ is called the tree growth of $G$ at $m$. Let $u$ and $v$ be endvertices of $G$, let $\left(\cdots, u_{-2}, u_{-1}, u_{0}, u_{1}\right.$, $\left.u_{2}, \cdots\right)$ and $\left(\cdots, v_{-2}, v_{-1}, v_{0}, v_{1}, v_{2}, \cdots\right)$ be the two-way infinite paths in $G_{u}$ and $G_{v}$ respectively, and let $B_{u, i}$ and $B_{v, i}$ be the tree growths in $G_{u}$ and $G_{v}$ at $u_{i}$ and $v_{i}$ respectively. We say that $G_{u}$ and $G_{v}$ are properly paired if either

$$
\begin{aligned}
B_{u, i} & \cong B_{v, i} \text { for } i \neq j, k, \\
\left|B_{v, k}\right| & =\left|B_{u, k}\right|-1 \text { and }\left|B_{u, j}\right|=\left|B_{v, j}\right|-1
\end{aligned}
$$




$$
B_{u, i} \cong B_{v, i} \text { for } i \neq j=k \text {, and }\left|B_{u, k}\right|=\left|B_{v, k}\right| \text {. }
$$

Clearly $G_{u}$ and $G_{v}$ have at least one proper pairing since $G$ induces one in the natural way.

Since $G$ has infinitely many endvertices, there are endvertices $u, v \in V(G)$ such that the naturally induced proper pairing of $G_{u}$ and $G_{v}$ is as in (1). Thus we can label the two-way infinite path in $G$ as $(\cdots,-2,-1,0,1,2, \cdots)$ so that, denoting by $B_{2}$ the tree growth of $G$ at vertex $i, B_{i} \cong B_{v, i}$ for $i \neq k$ and $\left|B_{v, k}\right|=\left|B_{k}\right|-1$. Therefore, $G$ can be obtained in either of two ways: from $G_{v}$ by replacing $B_{v, k}$ by $B_{u, k}$ or from $G_{u}$ by replacing $B_{u, j}$ by $B_{v, j}$. If this is the only proper pairing of $G_{u}$ and $G_{v}$, then the $\left\{G_{w}\right\}_{w \in V(G)}$ determine $G$ uniquely, up to isomorphism, and we have $G \cong H$.

So suppose there is another proper pairing of $G_{u}$ and $G_{v}$. There are two possibilities:

(A) $G_{u}$ was reflected with respect to $G_{v}$, that is, there is an $r>$ 0 such that either

(i) $B_{v, r-i} \cong B_{u, r+i+1}$ for all but at most two values of $i$ (these values correspond to the vertices at which corresponding tree growths might not be isomorphic), or

(ii) $B_{v, r-i} \cong B_{u, r+\imath}$ for all but at most two values of $i$. (Case (i) corresponds to the reflection taking place about the "midpoint" of the edge $\left(u_{r}, u_{r+1}\right)$ while case (ii) corresponds to the reflection taking place about the vertex $u_{r}$ ), or

(B) $G_{u}$ was translated with respect to $G_{v}$, that is, there is a $d \neq$ 0 such that

$$
B_{u, i} \cong B_{u, 2+d} \text { for all but at most two values of } i \text {. }
$$

Without loss of generality we may assume that $d>0$.

Case (A). Since subcases (i), (ii) are similar we shall consider only (ii). If $n=r-i$, then $i=r-n$ and so $r+i=2 r-n$. Hence, combining the proper pairings of $G_{u}$ and $G_{u}$ as in (1) and (A)-(ii) we have

$B_{n} \cong B_{u, n} \cong B_{v, 2 r-n} \cong B_{2 r-n}$ for all but a finite number of values of $n$.

A pivot of $G$ is a vertex $t$ of the two-way infinite path of $G$ such that $B_{n} \cong B_{2 t-n}$ for all but a finite number of values of $n$. Therefore, $r$ is a pivot of $G$ and there is no loss of generality in taking $r=0$. Thus we have

$B_{n} \cong B_{-n}$ for all but a finite number of values of $n$.

If $G$ has two pivots then we clearly have case (B) (to follow), so we can assume that the vertex 0 is the only pivot of $G$. It fol- 
lows that each $G_{v}, v$ an endvertex of $G$, has a unique pivot, identical to the pivot of $G$. A non-reflecting pair is a pair $(n,-n)$ with $B_{n} \not$ $B_{-n}$. Let

$$
p(G)=\max _{v}\left\{\text { number of non-reflecting pairs in } G_{v}\right\} .
$$

Any $G_{v}$ with $p(G)$ non-reflecting pairs must contain all non-reflecting pairs of $G$. Choose two such, $G_{v}$ and $G_{w}$, where $G_{w}$, say, has a non-reflecting pair $(n,-n)$ such that $n>k$ for all non-reflecting pairs $(k,-k)$ of $G_{v}$. $G$ is obtained from $G_{w}$ by replacing the smaller of $B_{w, n}$ and $B_{w,-n}$ by the larger. Hence $G \cong H$.

Case (B). Combining the proper pairings of $G_{u}$ and $G_{v}$ as they occur in (1) and (B) we have $B_{n} \cong B_{v, n} \cong B_{v, n+d} \cong B_{n+d}$ for all but a finite number of values of $n$, that is,

$$
B_{n} \cong B_{n+d} \text { for all but a finite number of values of } n \text {. }
$$

Since $G$ has an infinite number of endvertices, $B_{n} \cong B_{n+d}$ for all $n$ if and only if, for each endvertex $v, B_{v, n-d} \not B_{v, n} \not B_{v, n+d}$ for exactly one value of $n$. This situation can be recognized and $G$ is obtained from any such $G_{v}$ on replacing $B_{v, n}$ by $B_{v, n+d}$. Hence in this case $G \cong H$.

In all other cases we can define

$$
\begin{aligned}
& l=l(G)=\min \left\{n: B_{n} \not B_{n-d}\right\}, \\
& r=r(G)=\max \left\{n: B_{n} \not B_{n+d}\right\} .
\end{aligned}
$$

This time we define the centre of $G$ to be the subgraph of $G$ that is the union of the path between $l(G)$ and $r(G)$ and all tree growths rooted at vertices of this path. The length of the centre is $\mid l(G)-$ $r(G) \mid$. There are three possibilities: (i) $l(G)<r(G)$; (ii) $l(G)>r(G)$; or (iii) $l(G)=r(G)$.

Since $G$ has an infinite number of endvertices, $G$ has three endvertices $v_{1}, v_{2}, v_{3}$ such that $G_{v_{1}}, G_{v_{2}}$, and $G_{v_{3}}$ have different, finite, centre lengths. Let $e^{\prime}=\max _{i}$ \{centre length of $\left.G_{v_{i}}\right\}$ and let $e=\max \left\{e^{\prime}, d\right\}$. We claim that the centre length of $G$ is at most $e$.

In case (i), $G_{v}$ has centre length at least as great as $G$ if $v \in B_{l}$ or $v \notin B_{r}$. Also there is a constant $c_{l}$ such that, for each vertex $v \in B_{l}$, the centre length of $G_{v}$ is either $c_{l}$ or the centre length of $G$. Likewise there is a constant $c_{r}$ such that for each endvertex $v \in B_{r}$, the centre length of $G_{v}$ is either $c_{r}$ or the centre length of $G$. Thus, since the $G_{v_{i}}$ 's have different centre lengths, the centre length of $G$ is at most $e^{\prime}$.

In case (ii) the centre length of $G$ is at most $d$; for otherwise $d+r<l$ and so $B_{r} \cong B_{r+d}$, contradicting the definition of $r(G)$. 
In case (iii) the centre length of $G$ is zero.

Since there are only a finite number of isomorphism types of branch we see that, for any endvertex $v$,

$$
\left.\max _{k}\left\{\text { order of } B_{k}\right\} \leqq 1+\max _{k} \text { forder of } B_{v, k}\right\}=f<\infty .
$$

Let us call an endvertex $u_{1}$ of a 1-coherent graph $C$ a possible end of $C$ if the tree growths $\left(B_{k}^{\prime}\right.$ at $u_{k}$ ) on the (unique) one-way infinite path $\left(u_{1}, u_{2}, u_{3}, \cdots\right)$ starting at $u_{1}$ all have order at most $f$. Now let $v$ be a cut-vertex of $G$ such that $G_{v}$ has a 1-coherent component $C$ with the property that for each possible end $u_{1}$ of $C$ the largest $i\left(=k\right.$ say) for which $B_{i}^{\prime} \not B_{i+d}^{\prime}$ (in the above notation) is at least $2 d+e+f$. (Such a $v$, far enough away from the centre, certainly exists.) Suppose that $w_{1} \in V(C)$ is adjacent to $v$ in $G$ and that $\left(w_{1}, w_{2}, \cdots\right)$ is the one-way infinite path in $C$ starting at $w_{1}$ (and hence a section of the two-way infinite path $(\cdots,-2,-1,0,1,2, \cdots)$ of $G$ ). Then clearly, for some integer $t, u_{f}=w_{t}$. It follows that $\left(u_{f}, u_{f+1}, \cdots\right)$ is a section of the two-way infinite path of $G$, that $u_{k}$ is either $l(G)$ or $r(G)$, say $r(G)$, and that $l(G)=u_{j}$ for some $j, k-e \leqq$ $j \leqq k+e$. Since $j-f \geqq 2 d, G$ is obtained from $C$ by continuing indefinitely the pattern identifiable between $l(G)=u_{j}$ and $u_{f}$. Hence $G \cong H$.

Acknowledgment. We wish to express our thanks to Professor C. St. J. A. Nash-Williams for several valuable comments.

\section{REFERENCES}

1. J. Fisher, A counterexample to the countable version of a conjecture of Ulam, J. Combinatorial Theory, 7 (1969), 364-365.

2. J. Fisher, R. L. Graham, F. Harary, and J. A. B. Zonker, A simpler counterexample to the reconstruction conjecture for denumerable graphs, J. Combinatorial Theory, to appear.

3. F. Harary, Graph Theory, Addison-Wesley, Reading, Mass., 1969.

4. F. Harary, A. Schwenk and R. L. Scott, On the reconstruction of countable forests, preprint.

5. P. J. Kelly, A congruence theorem for trees, Pacific J. Math., 7 (1957), 961-968.

6. C. St. J. A. Nash-Williams, Infinite graphs-a survey, J. Combinatorial Theory, 3 (1967), 286-301.

7. S. M. Ulam, A Collection of Mathematical Problems, Wiley, New York, 1960.

Received March 1, 1972 and in revised form October 17, 1973.

UNIVERSITY OF WATERLOO, CANADA

AND

VANDERBILT UNIVERSITY 


\section{PACIFIC JOURNAL OF MATHEMATICS}

EDITORS

RICHARD ARens (Managing Editor)

University of California

Los Angeles, California 90024

\section{R. A. Beaumont}

University of Washington

Seattle. Washington 98105

\section{J. DugundjI}

Department of Mathematics University of Southern California Los Angeles, California 90007

D. Gilbarg and J. Milgram

Stanford University

Stanford. California 94305

\section{ASSOCIATE EDITORS}
E. F. BECKENBACH
B. H. NEUMANN
F. WOLF
K. Yoshida

\section{SUPPORTING INSTITUTIONS}

\author{
UNIVERSITY OF BRITISH COLUMBIA \\ CALIFORNIA INSTITUTE OF TECHNOLOGY \\ UNIVERSITY OF CALIFORNIA \\ MONTANA STATE UNIVERSITY \\ UNIVERSITY OF NEVADA \\ NEW MEXICO STATE UNIVERSITY \\ OREGON STATE UNIVERSITY \\ UNIVERSITY OF OREGON \\ OSAKA UNIVERSITY
}

\author{
UNIVERSITY OF SOUTHERN CALIFORNIA \\ STANFORD UNIVERSITY \\ UNIVERSITY OF TOKYO \\ UNIVERSITY OF UTAH \\ WASHINGTON STATE UNIVERSITY \\ UNIVERSITY OF WASHINGTON

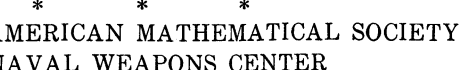

The Supporting Institutions listed above contribute to the cost of publication of this Journal, but they are not owners or publishers and have no responsibility for its content or policies.

Mathematical papers intended for publication in the Pacific Journal of Mathematics should be in typed form or offset-reproduced, (not dittoed), double spaced with large margins. Underline Greek letters in red, German in green, and script in blue. The first paragraph or two must be capable of being used separately as a synopsis of the entire paper. Items of the bibliography should not be cited there unless absolutely necessary, in which case they must be identified by author and Journal, rather than by item number. Manuscripts, in duplicate if possible, may be sent to any one of the four editors. Please classify according to the scheme of Math. Rev. Index to Vol. 39. All other communications to the editors should be addressed to the managing editor, or Elaine Barth, University of California, Los Angeles, California, 90024.

100 reprints are provided free for each article, only if page charges have been substantially paid Additional copies may be obtained at cost in multiples of 50 .

The Pacific of Journal Mathematics is issued monthly as of January 1966. Regular subscription rate: $\$ 72.00$ a year (6 Vols., 12 issues). Special rate: $\$ 36.00$ a year to individual members of supporting institutions.

Subscriptions, orders for back numbers, and changes of address should be sent to Pacific Journal of Mathematics, 103 Highland Boulevard, Berkeley, California, 94708.

\section{PUBLISHED BY PACIFIC JOURNAL OF MATHEMATICS, A NON-PROFIT CORPORATION}

Printed at Kokusai Bunken Insatsusha (International Academic Printing Co., Ltd.), 270, 3-chome Totsuka-cho. Shinjuku-ku, Tokyo 160. Japan.

Copyright (C) 1973 by Pacific Journal of Mathematics Manufactured and first issued in Japan 


\section{Pacific Journal of Mathematics}

\section{Vol. 52, No. $2 \quad$ February, 1974}

Harm Bart, Spectral properties of locally holomorphic vector-valued functions .....

J. Adrian (John) Bondy and Robert Louis Hemminger, Reconstructing infinite

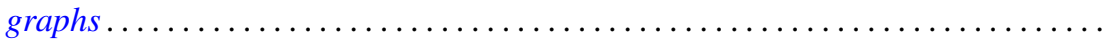

Bryan Edmund Cain and Richard J. Tondra, Biholomorphic approximation of planar

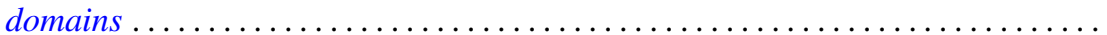

Richard Carey and Joel David Pincus, Eigenvalues of seminormal operators,

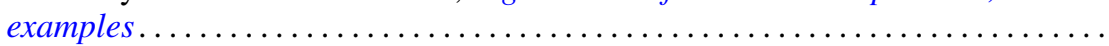

Tyrone Duncan, Absolute continuity for abstract Wiener spaces . . . . . . . . . . . . Joe Wayne Fisher and Louis Halle Rowen, An embedding of semiprime

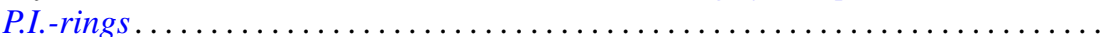

Andrew S. Geue, Precompact and collectively semi-precompact sets of semi-precompact continuous linear operators. . . . . . . . . . . . . . . .

Charles Lemuel Hagopian, Locally homeomorphic $\lambda$ connected plane continua ..... . Darald Joe Hartfiel, A study of convex sets of stochastic matrices induced by

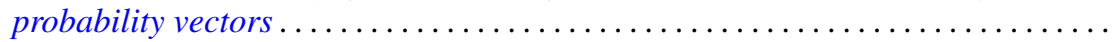

Yasunori Ishibashi, Some remarks on high order derivations $\ldots \ldots \ldots \ldots \ldots \ldots \ldots$ Donald Gordon James, Orthogonal groups of dyadic unimodular quadratic forms.

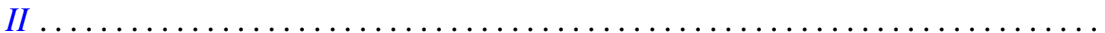

Geoffrey Thomas Jones, Projective pseudo-complemented semilattices . . . . . . . . . Darrell Conley Kent, Kelly Denis McKennon, G. Richardson and M. Schroder,

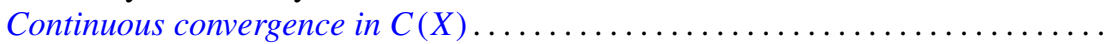

J. J. Koliha, Some convergence theorems in Banach algebras ...

Tsang Hai Kuo, Projections in the spaces of bounded linear oper

George Berry Leeman, Jr., A local estimate for typically real functions . .

475

Andrew Guy Markoe, A characterization of normal analytic spaces by the

homological codimension of the structure sheaf .........

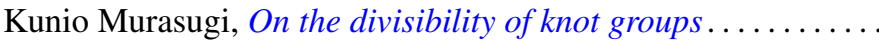

John Phillips, Perturbations of type I von Neumann algebras.

Billy E. Rhoades, Commutants of some quasi-Hausdorff matrices . .

David W. Roeder, Category theory applied to Pontryagin duality

Maxwell Alexander Rosenlicht, The nonminimality of the differential closure .

Peter Michael Rosenthal, On an inversion theorem for the general Mehler-Fock transform pair.

Alan Saleski, Stopping times for Bernoulli automorphisms

John Herman Scheuneman, Fundamental groups of compact complete locally affine complex surfaces. II. ........................

Vashishtha Narayan Singh, Reproducing kernels and operators with a cyclic vector. I. .

Peggy Strait, On the maximum and minimum of partial sums of random variables.

J. L. Brenner, Maximal ideals in the near ring of polynomials modulo 2 .

Ernst Gabor Straus, Remark on the preceding paper: "Ideals in near rings of polynomials over a field" ..........................

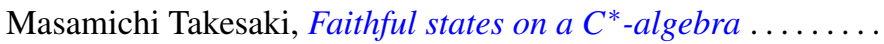

R. Michael Tanner, Some content maximizing properties of the regular simplex.

Andrew Bao-hwa Wang, An analogue of the Paley-Wiener theorem for certain

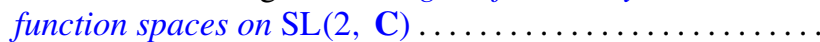

